\title{
The proposal of a clinical protocol to assess central and peripheral fatigue in Myotonic Dystrophy type 1
}

\author{
S. BALDANZI, G. RICCI, M. BOTTARI, L. CHICO, C. SIMONCINI, S. GABRIELE \\ Department of Clinical and Experimental Medicine, University of Pisa, Pisa, Italy.
}

\begin{abstract}
A B S T R A C T
DM1 is an autosomal-dominant disorder characterized by muscle weakness, myotonia, and multisystemic involvement. According to current literature muscle weakness and fatigue are among the main symptoms of DM1. Oxidative stress has been proposed to be one of the pathogenic factors of fatigue consequent to DM1. In this study, we investigated the dimensions of experienced and physiological fatigue in a sample of 26 DM1 patients (17 males, 9 females, mean age 41.6 years, $S D \pm 12.7$ ); experienced fatigue has been studied through Fatigue Severity Scale (FSS), and physiological fatigue was measured through an intermittent incremental exercise of the forearm muscles using a myometer; oxidative stress balance markers trend during aerobic exercise test have been collected. The occurrence of central fatigue in the sample means that central activation worsens during the motor contraction; interestingly FSS score was significantly correlated to MVC (before and after the effort, $r$-before $=-0.583, p<$ $0.01, r$-after $=-0.534, p<0.05)$, and to motor disability measured by MRC $(r=-0.496, p<0.05)$; moreover we found a strong tendency towards significance in the association to lactate baseline $(r=0.378, p=0.057)$. Results are discussed to define whether or not, based on clinical and laboratory grounds, such exercise training protocol may be suitable for proper management of DM1 patients; proper assessment of fatigue should be included in algorithms for data collection in DM1 patient registries.
\end{abstract}

$$
\begin{gathered}
\text { Key words } \\
\text { Myotonic Dystrophy } \bullet \text { Fatigue } \bullet \text { Central } \bullet \text { Peripheral } \bullet \text { Exercise }
\end{gathered}
$$

\section{Introduction}

Myotonic dystrophy type 1 (DM1), or "Steinert's disease", is an inherited multisystemic disorder caused by an unstable expansion of cytosine thymine - guanine (CTG) repeat in the non-translated region of the dystrophia myotonica-protein kinase gene (DMPK) (Huang \& Kuo 2005; Jorde et al. 2006; Finsterer et al. 2007); the mutation causes mis-splicing of mRNA species which can affect many cellular processes in different organs and tissues (Udd, 2012). Longer CTG repeat expansions are frequently associated with a more disabling phenotype but there is still not final agreement about this complex correlation (Udd \& Krahe 2012). The most common clinical features of DM1 phenotype include progressive muscular weakness, myotonia, cardiac arrhythmias, respiratory failures, daytime sleepiness and cognitive disorders (Harper, 2001; van Engelen 2015).

Although fatigue is a major condition in many chronic and progressive physical illnesses, it is reported to be more common in DM1 than in other neuromuscular diseases (Laberge et al. 2009) and most patients complain muscle fatigue since the early stages of disease even in absence of a strong correlation with the objective motor disability (Kalkman et al. 2005). Fatigue can be defined as the inability to maintain the expected level of motor performance over time (Allen et al. 2008), but its pathophysiology remains unclear (Kalkmann et al. 2005, Boerio et al. 2012).

Fatigue associated with motor performance has been considered to consist of two components: central and 
peripheral. Central fatigue is a progressive reduction in voluntary muscle activation during exercise, related to spinal or supraspinal factors (Gandevia, 2001; Taylor and Gandevia, 2008), while peripheral fatigue results from an altered neuromuscular transmission or muscular dysfunction.

A study of isometric exercise using transcranial motor cortex stimulation by Taylor and co-workers (2006) provided evidence that central fatigue emanates upstream of the pre-motor cortex in the prefrontal areas of the brain associated with cognitive and behavioural function. Bray et al. (2012) suggested that in healthy subjects the decrease in maximal force production observed during repeated maximal voluntary contraction (MVC) can be caused by a central mechanism, specifically the expenditure of CNS resources.

These considerations suggest that further studies on parallel CNS and muscle involvement could be useful to define the fatigability in DM1 patients.

Muscle fatigue could be due to coupling excitementcontraction, to lack of availability of substrates or blood flow and lack of adaptation of vasodilatation by nitric oxide (NO), and to the possible modifications of the intracellular environment and disruption of contractile apparatus (Heydemann et al. 2009).

Oxidative stress has been proposed to be one of the pathogenic factors of fatigue consequent to DM1 (Angelini and Tasca, 2012). Some studies linking nitric oxide species (NOS) and myotonic dystrophy suggests that although they are required for induction of dystrophia myotonica protein kinase (DMPK) expression during myogenesis, there is only a weak functional implication for such a generation and subsequent maintenance of DMPK expression (Carrasco et al. 2002).

Despite the fact that fatigue and exercise-induced fatigue are prominent in patients with DM1, limiting daily living activities and thereby affecting quality of life, this phenomenon remains poorly understood. Our aim has been then to get an overview of central and peripheral components of fatigue in a sample of patients meeting both genetic and clinical criteria for DM1, through a specifically designed multidimensional protocol.

\section{Methods}

Twenty-six patients with clinical and genetic diagnosis of DM1, according the International Consortium for myotonic dystrophies guidelines
(Moxley et al. 2002), referred to Neurological Clinic of University of Pisa, were recruited: 17 men, 9 women; age range 19-67 yrs; $41.6 \pm 12.7$ years. The study was conducted according to the principles expressed in the Declaration of Helsinki and written information about the purpose of the study was provided to all the patients before giving their consent.

Patients affected by serious psychiatric comorbidities were excluded.

Clinical and demographic characteristics of the patient sample are shown in Table 1.

The experimental protocol was displayed into three examination sessions:

a) comprehensive clinical evaluation;

b) behavioural assessment of experienced fatigue through the administration of specific questionnaires and clinical scales;

c) intermittent handgrip exercise protocol.

a) Neurological examination included clinical history collection and evaluation of muscle involvement, scored by Medical Research Council scale (MRC, Medical Research Council, 1943). Motor impairment was then assessed through Muscular Impairment Rating Scale (MIRS, Mathieu et al. 2001).

All patients were sub-grouped on the basis of CTG expansion size (Table 1). A complete assessment of extra-muscular clinical features was also performed, including evaluation of cardiac, respiratory, gastrointestinal, ocular and endocrinological involvement.

b) Behavioural assessment.

The assessment of experienced fatigue was performed by a trained psychologist, blinded to clinical examinations, in a quiet and comfortable room.

\section{FSS-Fatigue Severity Scale}

Fatigue Severity Scale-FSS is one of the most common questionnaire used to assess the impact of fatigue on daily activities; it has showed good reliability in DM1 (Krupp 1989; Laberge 2005).

\section{Individualized Neuromuscular Quality} of Life, INQoL

In order to investigate the disease subjective experience, we adopted the Individualized Neuromuscular Quality of Life Questionnaire (INQoL, Sansone et al. 2010), a 10 items-structured interview, that captures patient's physical and 


\begin{tabular}{|c|c|c|}
\hline Patients Enrolled (clinical study) & 26 & \\
\hline Muscular exercise protocol & 20 & \\
\hline Age & Age range $19-67$ yrs, & \\
\hline Sex & 17 men, 9 women & \\
\hline [CTG]n El & $\mathrm{N}=4$ & $15.4 \%$ \\
\hline [CTG]n E2 & $\mathrm{N}=20$ & $76.9 \%$ \\
\hline [CTG]n E3 & $\mathrm{N}=2$ & $7.7 \%$ \\
\hline BMI & $24.9 \pm 5.63 \mathrm{~kg} / \mathrm{m} 2$ & \\
\hline Time from disease onset & $17.8 \pm$ SD 12.7 years & \\
\hline \multicolumn{3}{|l|}{$\begin{array}{l}\text { Severity of muscular (MIRS) (MIRS) } \\
\text { involvement (MIRS) }\end{array}$} \\
\hline MIRS=1 & $\mathrm{N}=0$ & \\
\hline MIRS=2 & $\mathrm{N}=12$ & $46.1 \%$ \\
\hline MIRS=3 & $N=10$ & $38.5 \%$ \\
\hline MIRS=4 & $\mathrm{N}=4$ & $15.4 \%$ \\
\hline MIRS=5 & $\mathrm{N}=0$ & \\
\hline
\end{tabular}

psychosocial limitations related to the muscle disease. Single domain scores and a total score are calculated in term of percentage: a higher percentage is indicative of a greater impact of the disease on quality of life.

\section{Cognitive assessment of attention abilities}

Sincemotorperformancealso requires theexpenditure of CNS processes, that tap into attentional resources, each participant underwent a cognitive assessment session focusing on attentional abilities, with the aim to identify patients affected by significant dysfunction likely to account for reduction in overall performance. A trained neuropsychologist administered the following protocol:

- trail Making Test (A and B tables, Reitan et al. 1958);

- $\quad$ stroop test (Lezak et al. 2012);

- digit span (Spinnler e Tognoni 1987).

\section{ESS-Epworth Sleepiness Scale}

The Epworth Sleepiness Scale (ESS), was developed to measure the general level of sleepiness, conceptually defined as sleep propensity (Johns 1991).Scores of 10 or higher are considered as indicative of excessive daytime sleepiness.

c) Intermittent handgrip exercise protocol.

A handgrip dynamometer (Multi-Myometer, MIE Medical research Ltd., Leeds, UK) was used to monitor and record muscle force generation (in Newtons: $\mathrm{N})$ during maximal voluntary handgrip contractions (MVC). Drawing from procedures described by Søgaard et al. (2006), and in order to prevent hand myotonia, participants performed a pre-test warm-up phase consisting of some (4-5) series of intermittent hand contractions using a foam rubber ball. Then, at 5-min intervals thereafter, participants completed a single MVC, each lasting 5-s in duration. The force produced during a 1-s window straddling the peak force value generated during each MVC squeeze was used for analyses. All contractions were performed while sitting at a table, with the forearm outstretched at $90^{\circ}$ elbow flexion and parallel to the sagittal plane in a static, neutral posture (i.e., positioned halfway between pronation and supination) with the elbow and base of the hand situated on a forearm/hand tracing superimposed on the flat surface of the table. Participants were given verbal instructions to "squeeze the handles as hard as possible for 5 seconds" prior to each MVC and verbal encouragement to "squeeze hard, hard, hard". The exercise was performed with the patient's dominant arm (Figure 1).

In order to standardize force production across a broad range of raw force production values (i.e., some people had much higher force production than others), patients were asked to make $3 \mathrm{MVC}$, with 5 minutes interval to recover and the mean MVC value was computed; recovery from warm-up was then carried out for 20 minutes. 
Forearm muscle exercise $(20 \%-40 \%-60 \%)$

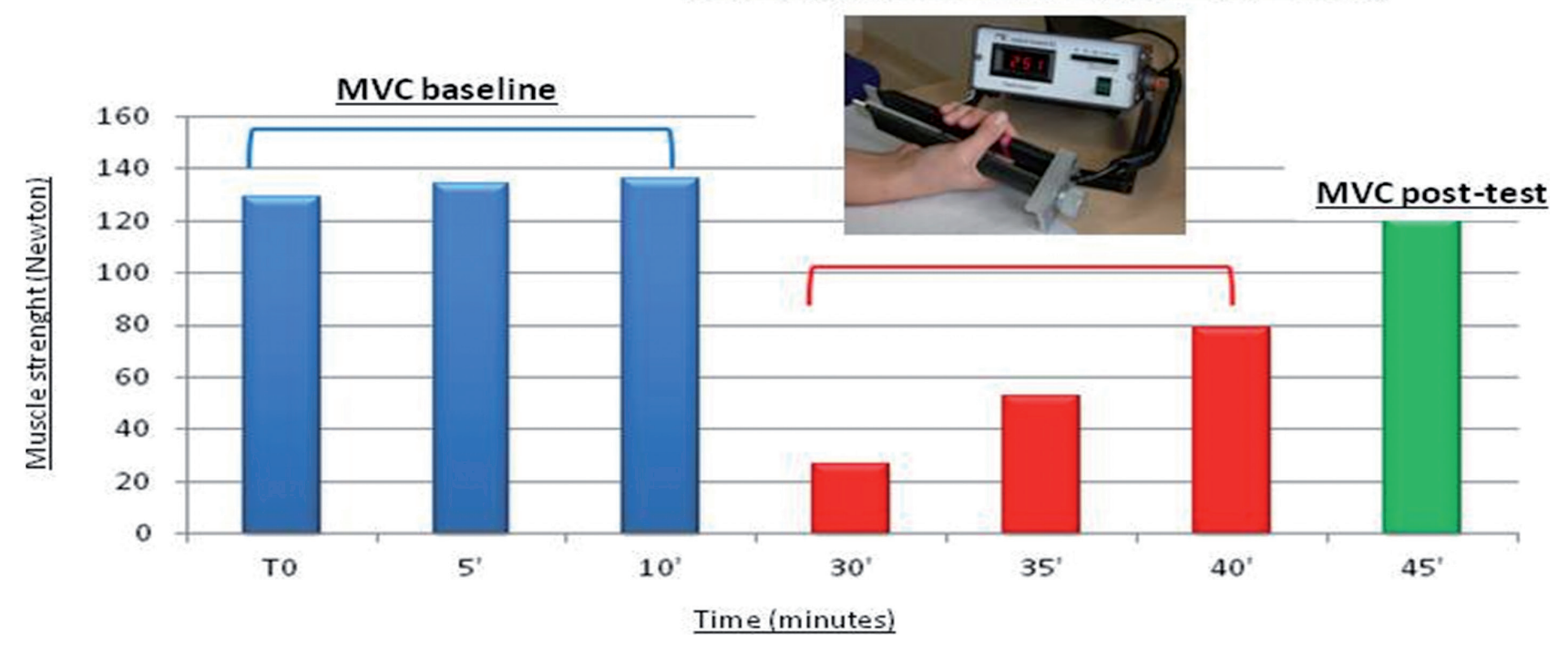

Fig. 1. - Study design diagram.

Blood sampling was performed before the beginning of the exercise protocol to determine baseline lactic acid (LA), creatine phosphokinase (CPK) and markers of oxidative stress (AOPP, FRAP, SH) levels.

The exercise protocol consisted of 3 trials of intermittent fatiguing contractions, lasting 1 minute each separated by 1 min of rest; the 3 trials were performed respectively at 20\%-40\%-60\% of individual mean MVC. During exercise, a visual feedback helped the subjects to maintain a steady contraction and full attention.

Blood sampling was then performed to assess LA, CPK and markers of oxidative stress (AOPP, FRAP, SH) levels immediately after the end of the exercise protocol. After 4' at rest, residual MVC (MVC_60\%) was determined through one single attempt of maximal strength lasting 5 '.

\section{Statistics}

Statistical analyses were carried out using the Statistical Package for Social Science (SPSS), version 21.0 and were performed by a medical statistician. In order to investigate the relationship between central and peripheric measures of fatigue, we computed the bivariate correlation coefficients, Pearson's coefficient for parametric variables and Spearman's coefficient for non parametric variables. Variables were compared between groups using the Pearson chi-square for categorical variables,
Student $\mathrm{t}$ test, the Mann-Whitney U test. The $\mathrm{p}$ value $<0.05$ was considered statistically significant. All statistical tests were two tailed.

\section{Results}

\section{Clinical assessment}

All patients $(\mathrm{N}=26)$ were molecularly defined and grouped on the basis of CTG repeat classes (Table 1). Mean time from disease onset was $17.8 \pm \mathrm{SD}$ 12.7 years; only one patient had a congenital onset of DM1.

Muscular impairment was assessed by MRC (Medical Research Council) and categorized Muscular Impairment Rating Scale (MIRS).

[CTG]n expansion class was significantly associated to muscle impairment measured by MIRS ( $\mathrm{r}=$ $-0.439 ; \mathrm{p}<0.05)$ and by MRC $(\mathrm{r}=-0.439 ; \mathrm{p}<0.05)$; moreover MRC was negatively related to patients age $(\mathrm{r}=-0.425 ; \mathrm{p}<0.05)$, and positively to $\mathrm{MVC}_{-}$ baseline and MVC_60\% ( $r=0.640$ and $r=0.584$ respectively, $\mathrm{p}<0,01)$.

Mean MRC score was 71.61 7,82 DS (Table 1).

[CTG]n genetic expansion show significant correlations to motor disability assessed by MIRS $(\mathrm{r}=0.640 ; \mathrm{p}<0.01)$ and by total MRC score $(\mathrm{r}=$ $-0.536 ; \mathrm{p}<0.01)$.

Patients' extramuscular involvement has been described in Table 2. 
Tab. 2. - Multisystemic features of recruited DM1 patients.

\begin{tabular}{|c|c|c|}
\hline Cardiological & 12 & $46.1 \%$ \\
\hline Respiratory & 11 & $42.3 \%$ \\
\hline Ocular & 7 & $26.9 \%$ \\
\hline Endocrinological & 9 & $34.6 \%$ \\
\hline Gastrointestinal & 12 & $46.1 \%$ \\
\hline Swallowing & 8 & $30.8 \%$ \\
\hline
\end{tabular}

included cardiac $(n=12,46.1 \% ; 3$ patients with pacemaker) and respiratory impairment ( $n=10,38.5 \%$; 8 patients using VMNI). Moreover $26.9 \%$ of patients had ocular impairment (mainly cataract); $34.6 \%$ had endocrine system impairment; $46.1 \%$ had gastrointestinal symptoms (prevalentemente colelitiasi ed alternanza stipsi-diarrea) and $30.8 \%$ had troubles with swallowing. Only one patient presented with an obstructive sleep apnea syndrome. (conteggiato nel respiratorio).

\section{Biochemical Parameters: lactic acid and markers of oxidative stress}

Under basal conditions lactic acid levels in DM1 patients resulted $12.665 \mathrm{mg} / \mathrm{dl} \pm 6.001$ (normal values $4.5-19.8 \mathrm{mg} / \mathrm{dl})$. At $60 \%$ of MVC lactic acid levels were $23.004 \pm 8.443 \mathrm{mg} / \mathrm{dl}$ : an increase of $81,63 \%$ respect to baseline was observed $(\mathrm{p}<0,001)$, which indicates that patients reached the anaerobic threshold. Baseline values of oxidative stress biomarkers have been compared with a healthy subjects control group of 15 subjects, matched for age and sex.

AOPP basal values resulted significantly higher in patients respect to healthy controls (patients: 375.95 $\pm 176.21 \mathrm{nmol} / \mathrm{ml}$; HC: $163.59 \pm 123.34 \mathrm{nmol} / \mathrm{ml}$; $\mathrm{p}=0,0002$ ); conversely no significant differences were found between and HC in FRAP and plasmatic thiols (FRAP in mmol/l, mean \pm sd: $0.7403 \pm$ 0.1367 ; plasmatic thiols in $\mu \mathrm{mol} / 1$, mean $\pm \mathrm{sd}$ : $0.3827 \pm 0.112)$ and $\mathrm{HC}($ FRAP in mmol/l, mean \pm sd: $0.801 \pm 0.104$; plasmatic thiols in $\mu \mathrm{mol} / \mathrm{l}$, mean \pm sd: $0.432 \pm 0.303$ ). At $60 \%$ of MVC oxidative stress biomarkers do not differ statistically from baseline.

\section{Perceived fatigue assessment}

Subjective perception of fatigue was assessed calculating FSS total score and single item score: mean total score (mean $\pm \mathrm{sd}$ ) was $4.8 \pm 0.2$, cut-off $=4.6$ (Krupp et al. 1989), (Figure 2). Total FSS score (43.6 \pm 12.4 ) is significantly associated to different clinical and physiological measures of fatigue. Single item analysis scores are shown in Figure 2. Statistical analysis revealed that total FSS score (mean $\pm \mathrm{sd}$ ) was $43.6 \pm 12.4$ and resulted significantly associated to: total MRC ( $\mathrm{r}=-0.496 ; \mathrm{p}=0.01)$; MVC_baseline $(\mathrm{r}=$ $-0.583 ; \mathrm{p}<0.01)$; MVC_60\% $(\mathrm{r}=-0.534 ; \mathrm{p}<0.05)$; mean patients age $(r=0.428 ; \mathrm{p}<0.05)$.
We found a strong tendency towards significance in the association between FSS total score and baseline lactic acid value $(\mathrm{r}=0.378 ; \mathrm{p}=0.057)$.

\section{Quality of life assessment}

Figure 3 shows the altered domains of quality of life detected by INQoL interview in DM1 patients (higher percentages correspond to higher disease impact on patients' QoL).

By considering the single INQoL Domain Scores, particularly item 1 and 4, we observed that the Fatigue domain (44.6\%) has high impact as well as the Weakness domain (49.3\%). Patients answers to item 1 of the INQoL ("Do you have any muscle weakness due to your muscle condition?") regarding subjective muscle weakness have been expressed on a 7-point Likert scale and correlate with [CTG]n (Spearman r $=0.459 ; \mathrm{p}<0.05)$ and with motor disability expressed by MIRS (Spearman $r=0.519$; $p<0.01$ ).

Patients answers to item 4 of the INQoL (" $D o$ you feel tired/fatigued as a result of your muscle condition?") do not show significant associations to any of the other variables.

\section{Attentional resources}

Cognitive assessment revealed that $46.2 \%$ of patients presents impaired attentional abilities while 50\% shows normal attentional functioning; one patient was not compliant to test administration (Figure 4).

\section{Daytime sleepiness evaluation}

Mean total ESS score in our sample was (mean \pm sd) $7.9 \pm 3.4$; only 6 patients $(23.1 \%)$ reached the cut-off score for daytime sleepiness diagnosis; ESS total and single item scores did not correlate to any other clinical parameter (Figure 5). 


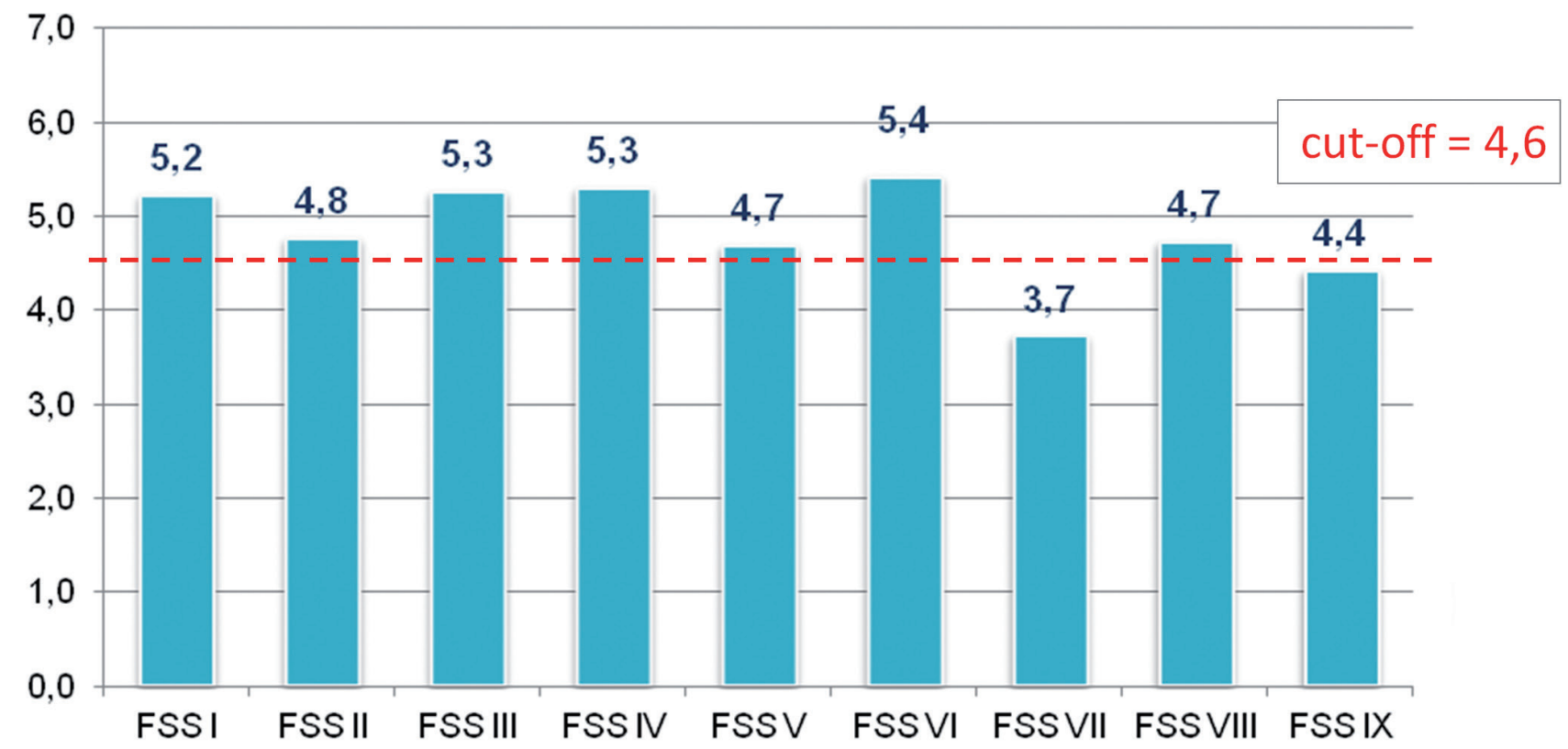

Fig. 2. - Central dimensions of fatigue in DM1 patients assessed by Fatigue Severity Scale (FSS) item analysis; FSS mean total score (mean $\pm \mathrm{sd}$ ) $4.8 \pm 0.2$.

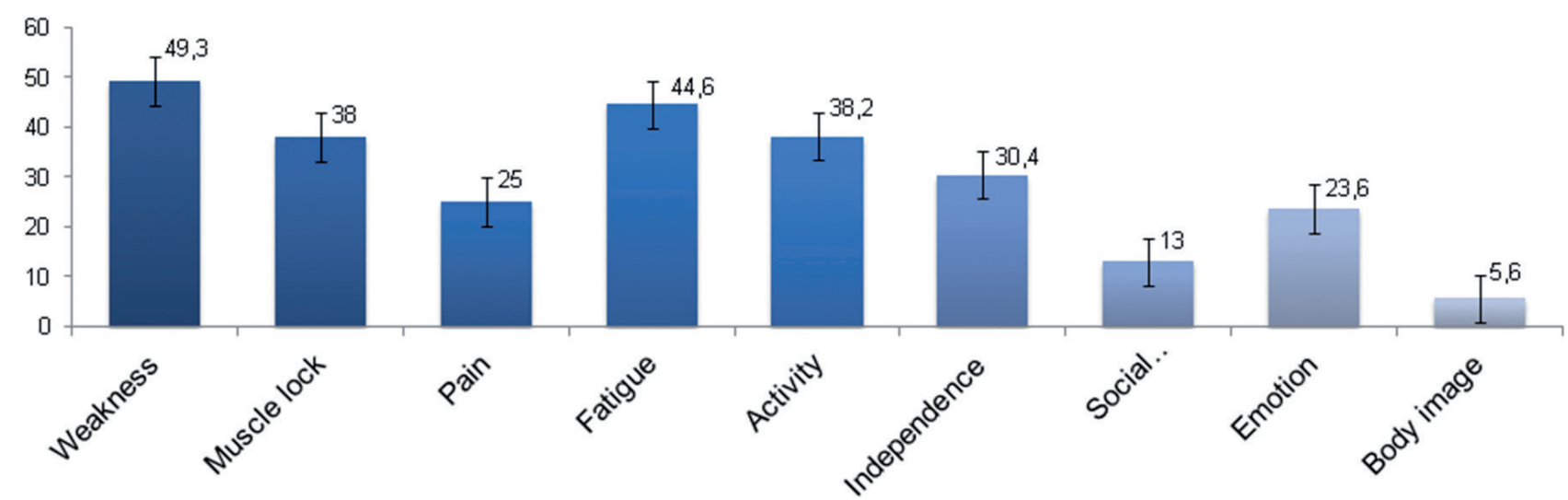

Fig. 3. - Quality of life assessment through INQoL interview of recruited DM1 patients, analyzing the impact of each symptom on various domains of everyday life; mean total INQoL score $=19,1 \%$.

\section{Muscular strength assessment through "hand-grip" exercise protocol}

Among the recruited patients, the "hand-grip" exercise protocol was performed by 20 subjects; 6 patients were not compliant to complete the exercise protocol.

The MVC was measured at baseline and at $60 \%$ of individual mean MVC in order to test response to exercise: mean ( \pm sd) MVC_baseline was $135.4 \pm 101.7$ and MVC_60\% was $120.9 \pm$ 100.9; after the exercise there was a statistically significant decrease (MVC_baseline - MVC_60\%) of maximum voluntary contraction $(\mathrm{p}<0,05)$ showing no statistically significant correlations with other variables investigated, except for age $(\mathrm{r}=-0.439$; $\mathrm{p}<$ 0.05), (Figure 6).

\section{Discussion}

Fatigue in DM1 is reported to be more common than in other neuromuscular diseases, even in absence of a clear association with the objective motor disability (Laberge et al. 2009). Fatigue substantially contributes to decrements in quality of 


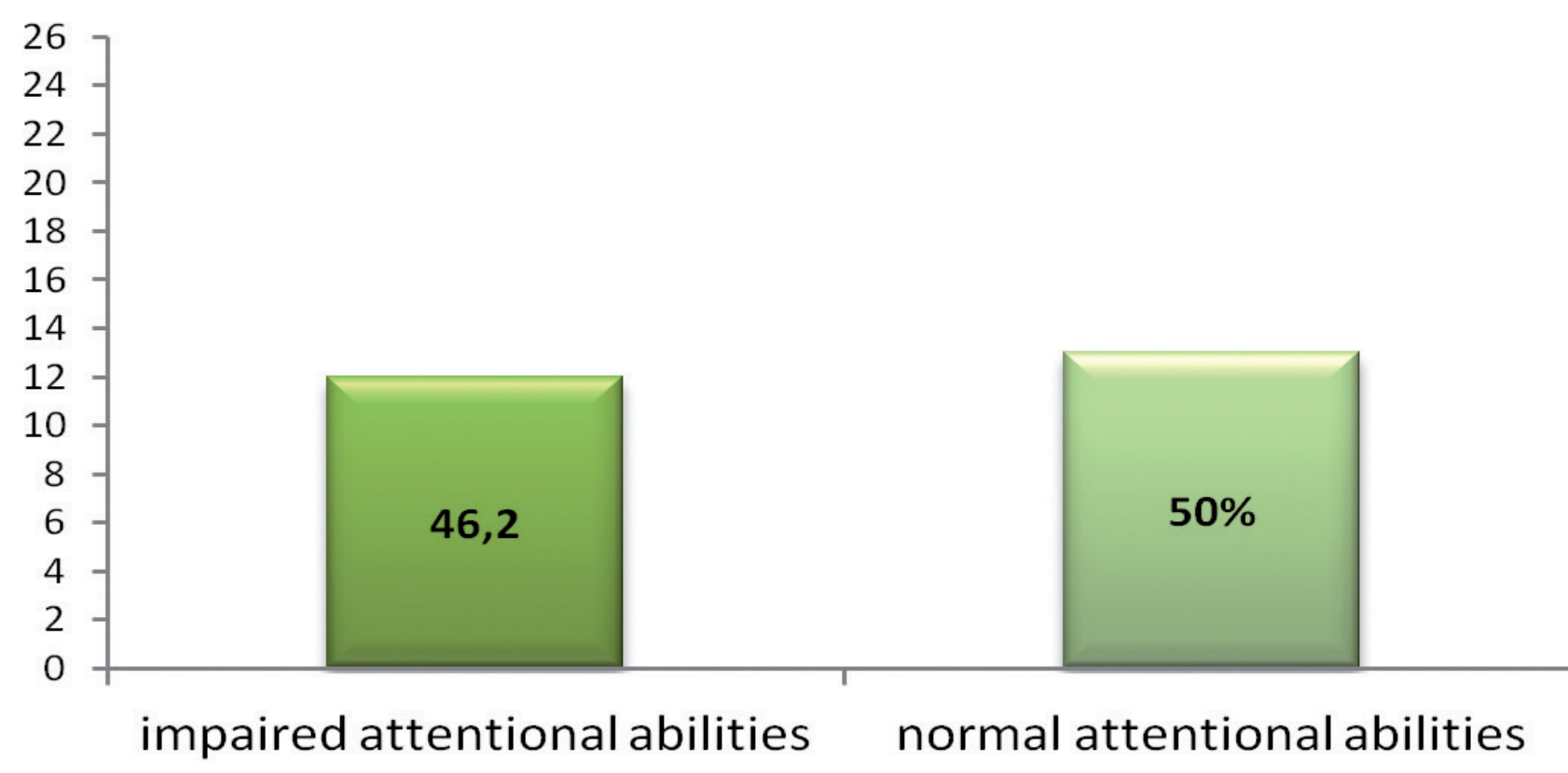

Fig. 4. - Percentages of DM1 patients affected by attentional dysfunction in the sample ( $N=13$ normal abilities; $N=12$ impaired abilities; $\mathrm{N}=1$ uncompliant).

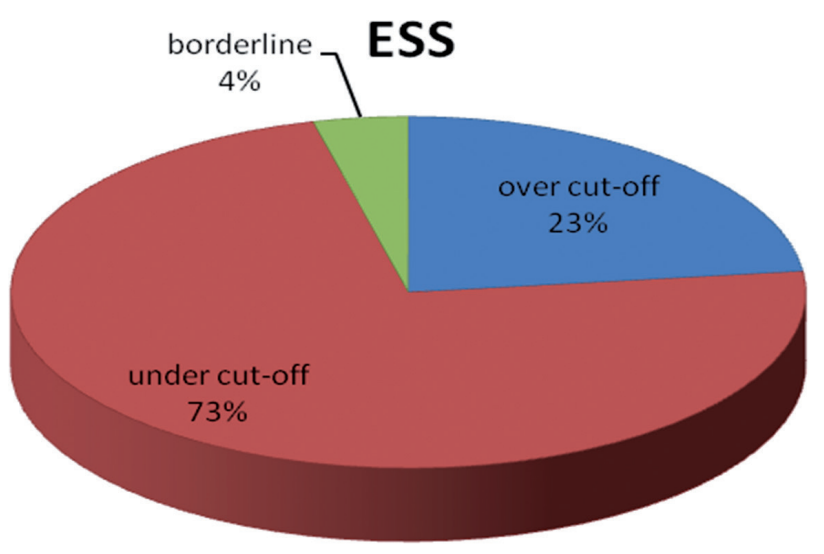

Fig. 5. - Prevalence of daytime sleepiness assessed by ESS scores.

life (Hermans et al. 2013, Ørngreen et al. 2005) but our understanding of the interaction between central and peripheral components is still limited and specific clinical rating tools have yet to be designed. Muscle fatigue is a multifactorial process and different neurobiological mechanisms could be involved in its pathogenesis: previous works have indicated alterations of sarcolemmal excitability, electro-mechanical coupling, availability of energy substrates, blood flow and adaptation exercise with vasodilation induced by nitric oxide (NO), as well as any modifications intracellular environment altering the contractile apparatus and oxidative stress as interplaying factors (Angelini \& Tasca 2012; Montes et al. 2011).

In order to address this issue we recruited 26 adult-onset DM1 patients able to perform a multidimensional evaluation protocol specifically designed to evaluate "experienced" and "physiological" components of fatigue; the present study has taken to a preliminary definition of a clinical protocol for the characterization of both fatigue components in DM1 patients.

We defined a simple handgrip exercise protocol consisted of intermittent fatiguing contractions in order to evaluate the muscle response; this kind of exercise is mainly aerobic at the beginning of the test and becomes gradually anaerobic as the effort grows, due to progressive recruitment of rapid motor units (Milner-Brown et al. 1973).

This protocol showed to be easily deployable in clinical settings and it resulted reliable in assessing the ongoing muscle fatigue process (Figure 6), as documented by the significant decrease in MCV at the end of the exercise. All patients reached the anaerobic threshold as showed by the increase in muscle lactic acid after the exercise: at $60 \%$ MVC the increase was about $81.6 \%$ respect to baseline.

Our protocol also includes the assay of peripheral blood oxidative stress parameters at baseline and during exercise but it fails to detect changes in 


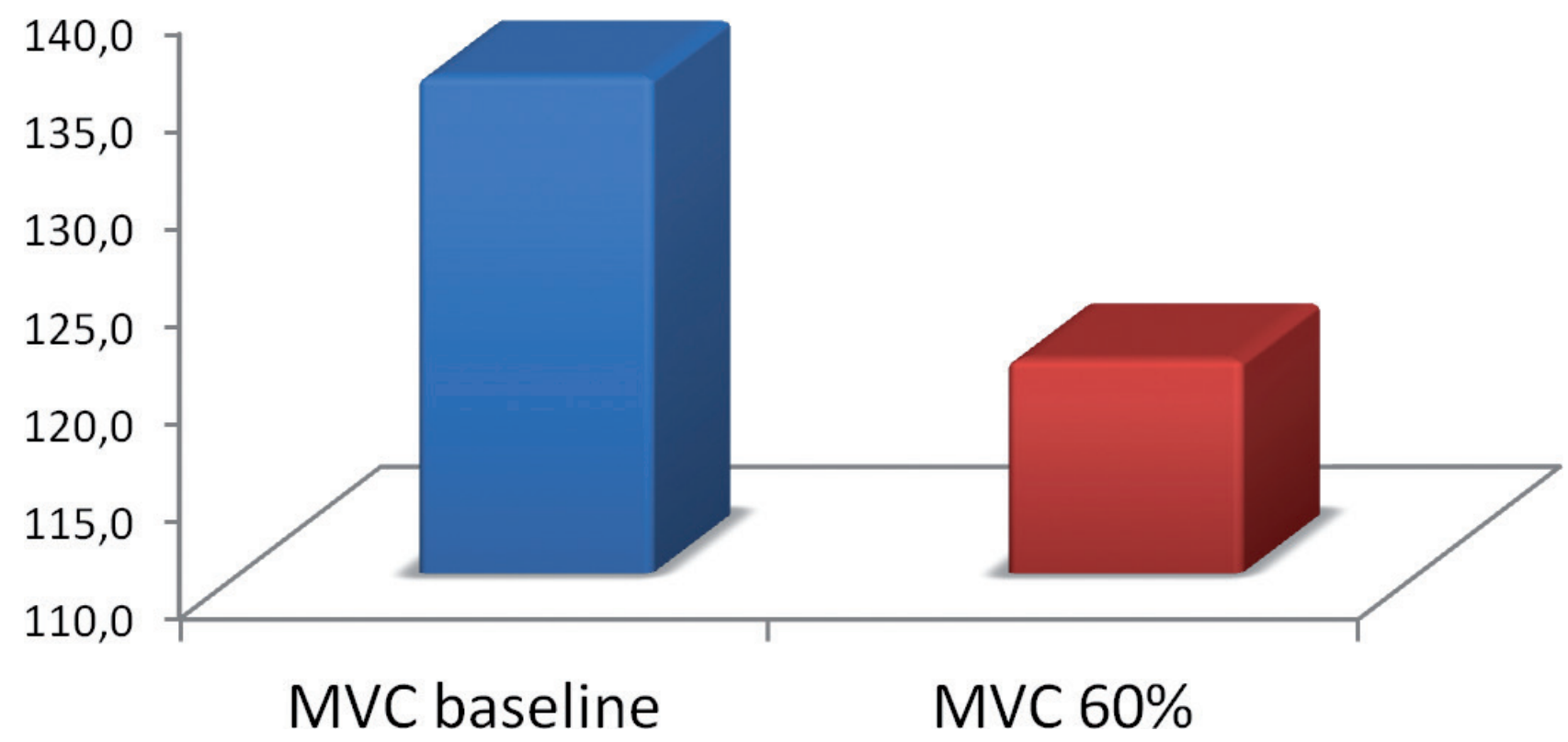

Fig. 6. - Changes in muscular strenght after $60 \%$ effort. MVC= maximum voluntary contraction. Mean values (Newton, N): MVC baseline =135.4 \pm 101.7 ; MVC 60\% = 120.9 \pm 100.9

oxidative balance. The abnormal CTG repetition, responsible for an altered DMPK function in DM1, leads to a compromised myofiber metabolic homeostasis, thus increasing the susceptibility of cells to oxidative stress, through the activation of different pathways of signal transduction, as postulated by several evidences in literature (Usuki et al. 2000; Pantic et al. 2013). Notably, among the pathophysiological hypotheses of muscle fatigue, a huge amount of data seem to suggest oxidative stress as one of the main mechanisms involved in the genesis of muscle fatigue (Reid, 2008).

The lack of statistically significant differences between the levels of non-enzymatic antioxidants of patients compared to controls in our study may depend upon an excessive production of ROS in DM1 patients, resulting in increase of oxidative damage (documented by higher AOPP baseline levels patients compared to healthy controls), that is likely to be counteracted thus by compensatory mechanisms increasing the antioxidant defense.

Oxidative stress parameters do not show significant changes at $60 \%$ MVC compared to baseline, consistently with the fact that the length of exercise protocol could not be sufficient to account for the activation of the biochemical pathway that leads to protein damage by ROS, as well as an improvement in the antioxidant defence mechanisms. Moreover in basal conditions muscular exercise naturally determines an immediate increase in ROS production with an increase in antioxidant defences too. We assume that a longer kinetic could be necessary to have appreciable changes in oxidative stress biomarkers that should be reassessed at later stages after the end of the exercise (2-6-9-24 hours). Even if only few studies addressed this topic in literature up to now, it is interesting to note that previous works adopting an electrophysiological approach, found that a submaximal fatiguing motor task was not able to further affect the cortical and neuromuscular properties that were already altered at baseline in DM1 patients (Boerio et al. 2012); this finding is in line with our results, and in accordance with a further study that has showed smaller changes in peripheral characteristics of fatigue in patients with DM1 compared to healthy subjects (Schillings et al. 2007). We can argue that adaptation of cortical and neuromuscular properties to baseline alteration may prevent patients from an enhanced fatigue after an exercise performed at a given percentage of MVC compared to controls.

Fatigue has been also been also evaluated from patients' subjective perspective and resulted associated with clinical parameters related to the underlying disease.

In particular, we observed an association between the degree of motor disability, assessed by MRC, and the total FSS score, indicating that patients 
with greater motor impairment also perceive greater fatigue. To this regard subjective experience of fatigue is also negatively associated to MVC, (both baseline and 60\%), and positively to baseline lactate. By administering INQoL questionnaire we assessed the impact of fatigue and muscular weakness on DM1 patinents' QoL. According to previous observations (Baldanzi et al. 2016, Peric et al. 2013, Meola and Sansone 2007), also in the present analysis INQoL total score revealed a mild impact of disease disability in DM1 patients. Statistical analysis showed an interesting association between subjective muscular weakness (item1), [CTG] $\mathrm{n}$ expansion and motor disability (MIRS). None of the other patient reported measures correlates with physiological parameters of muscle fatigue, indicating that probably this clinical scale has as the primary focus to detect specific data on the daily life of patients.

In our sample the statistical analysis of correlation between daytime sleepiness, measured by ESS, and fatigue, measured by FSS, was not significant, despite the fact that $23 \%$ of patients report a moderate degree of daytime sleepiness (Figure 5). We may suggest that attention disorders, detected in about half of the recruited patients, and low disease awareness (Baldanzi et al. 2016), interfere with patients' reliability to answer to the self-administered and to properly quantify the phenomenon of daytime sleepiness. In addition, muscle fatigue and daytime sleepiness show an a partially overlapping presentation (Hermans et al. 2013) that raise the need for validated and shared methodology to discriminate them.

It would be important to extend our study to a wider patients series to further investigate the relationship between functional assessment of the symptom "fatigue" and objective assessment by means of muscle exercise testing protocols. Moreover, the protocol has to be refined for a deeper understanding of the contribution of DM1-related brain dysfunction to motor performance: since a complete analysis of this phenomenon requires a combination of mental and physical demands, we should argue that a detailed evaluation of mood fluctuations could have been a helpful contribution to our study; future studies could aim to estimate to what extent cognitive and motor demands can interact with mood state, particularly considering the frequent occurrence of apathy in DM1 patients' samples.
The utility of a "Fatigue protocol" evaluating central as well as peripheral dimensions allows us to assume possible applications in the clinical evaluation of muscle fatigue in myotonic dystrophy, in the implementation of case report forms for disease registries, and as a reference for the evaluation of disease staging and trial readiness.

\section{Acknowledgements}

French Muscular Dystrophy Association (AFM), Grant\#16216.

\section{References}

Allen D.G., Lamb G.D., Westerblad H. Skeletal muscle fatigue: cellular mechanisms. Physiol Rev., 88 (1): 287-332, 2008.

Angelini C., Tasca E. Fatigue in muscular dystrophies. Neuromuscul Disord., 22: S214-20, 2012.

Balaban E., Centini C., Pompeiano P. Tonic gravity changes after gene expression in the efferent vestibular nucleus. NeuroReport, 13: 187-190, 2002.

Baldanzi S., Bevilacqua F., Lorio R., Volpi L., Simoncini C., Petrucci A., Cosottini M., Massimetti G., Tognoni G., Ricci G., Angelini C., Siciliano G. Disease awareness in myotonic dystrophy type 1 : an observational cross-sectional study. Orphanet $J$ Rare Dis., 11: 34, 2016.

Boërio D., Lefaucheur J.P., Bassez G., Hogrel J.Y. Central and peripheral components of exerciserelated fatigability in myotonic dystrophy type 1 . Acta Neurol Scand., 125: 38-46, 2012.

Bray S.R., Graham J.D., Martin Ginis K.A., Hicks A.L. Cognitive task performance causes impaired maximum force production in human hand flexor muscles. Biol Psychol., 89: 195-200, 2012.

Carrasco M., Canicio J., Palacín M., Zorzano A., Kaliman P. Identification of intracellular signaling pathways that induce myotonic dystrophy protein kinase expression during myogenesis. Endocrinology., 143: 3017-25, 2002.

Finsterer J., Stollberger C., Blazek G., Kunafer M., Prager E. Cardiac involvement over 10 years in myotonic and Becker muscular dystrophy and mitochondrial disorder. Int. J. Cardiol., 119: 176-184, 2007.

Flachenecker P., Kümpfel T., Kallmann B., Gottschalk M., Grauer O., Rieckmann P., Trenkwalder C., Toyka K.V. Fatigue in multiple sclerosis: a comparison 
of different rating scales and correlation to clinical parameters. Mult. Scler., 8 (6): 523-6, 2002.

Franzini M., Fornaciari I., Siciliano G., Volpi L., Ricci G., Marchi S., Gagliardi G., Baggiani A., Torracca F., Fierabracci V., Miccoli M., Pompella A., Emdin M., Paolicchi A. Serum gamma-glutamyltransferase fractions in myotonic dystrophy type I: differences with healthy subjects and patients with liver disease. Clin. Biochem., 43: 1246-8, 2010.

Gandevia S.C. Spinal and supraspinal factors in human muscle fatigue. Physiol. Rev., 81: 1725-89, 2001.

Heydemann A., McNally E. NO more muscle fatigue. J. Clin. Invest., 119 (3): 448-50, 2009.

Harper P.S. Major Problems in Neurology: Myotonic Dystrophy. London UK: W.B. Saunders; 2001.

Hermans M.C., Merkies I.S., Laberge L., Blom E.W., Tennant A., Faber C.G. Fatigue and daytime sleepiness scale in myotonic dystrophy type 1 . Muscle Nerve., 47: 89-95, 2013.

Huang C.C., Kuo H.C. Myotonic dystrophies. Chang Gung Med J., 28 (8): 517-26, 2005.

Jorde L., Carey J., Bamshad M., White R. Medical Genetics. St Louis: Mosby, 80-81, 2006.

Johns M.W. A new method for measuring daytime sleepiness: the Epworth Sleepiness Scale. Sleep, 14: 540-545, 1991.

Kalkman J.S., Schillings M.L., Zwarts M.J., van Engelen B.G., Bleijenberg G. The development of a model of fatigue in neuromuscular disorders: a longitudinal study. J. Psychosom. Res., 62: 571-9, 2007.

Kalkman J.S., Schillings M.L., van der Werf S.P., Padberg G.W., Zwarts M.J., van Engelen B.G., Bleijenberg G. Experienced fatigue in facioscapulohumeral dystrophy, myotonic dystrophy, and HMSN-I. J. Neurol. Neurosurg. Psychiatry., 76: 1406-9, 2005.

Krupp L.B., LaRocca N.G., Muir-Nash J., Steinberg A.D. The fatigue severity scale. Application to patients with multiple sclerosis and systemic lupus erythematosus. Arch. Neurol., 46: 1121-3, 1989.

Kuwahara H., Horie T., Ishikawa S., Tsuda C., Kawakami S., Noda Y., Kaneko T., Tahara S., Tachibana T., Okabe M., Melki J., Takano R., Toda T., Morikawa D., Nojiri H., Kurosawa H., Shirasawa T., Shimizu T. Oxidative stress in skeletal muscle causes severe disturbance of exercise activity without muscle atrophy. Free Radic. Biol. Med., 48: 1252-62, 2010.

Laberge L., Gagnon C., Jean S., Mathieu J. Fatigue and daytime sleepiness rating scales in myotonic dystrophy: a study of reliability. J. Neurol. Neurosurg. Psychiatry., 76: 1403-5, 2005.

Laberge L., Dauvilliers Y., Bégin P., Richer L., Jean S., Mathieu J. Fatigue and daytime sleepiness in patients with myotonic dystrophy type 1: to lump or split? Neuromuscul. Disord., 19: 397-402, 2009.

Leelarungrayub D., Khansuwan R., Pothongsunun P., Klaphajone J. N-acetylcysteine supplementation controls total antioxidant capacity, creatine kinase, lactate, and tumor necrotic factor-alpha against oxidative stress induced by graded exercise in sedentary men. Oxid. Med. Cell. Longev., 2011: 329643, 2011.

Lezak M.D., Howieson D.B., Bigler E.D., Tranel D. Neuropsychological Assessment, 5th edn. Oxford, UK: Oxford University Press, 2012.

Mathieu J., Boivin H., Meunier D., Gaudreault M., Bègin P. Assessment of a disease-specific muscular impairment rating scale in myotonic dystrophy. Neurology, 56: 336-40, 2001.

Meola G., Sansone V. Cerebral involvement in myotonic dystrophies. Muscle Nerve, 36: 294-306, 2007.

Milner-Brown H.S., Stein R.B., Yemm R. Changes in firing rate of human motor units during linearly changing voluntary contractions. J. Physiol., 230: 371-390, 1973.

Montes J., Dunaway S., Montgomery M.J., Sproule D., Kaufmann P., De Vivo D.C., Rao A.K. Fatigue leads to gait changes in spinal muscular atrophy. Muscle Nerve., 43: 485-8, 2011.

Moxley R.T. 3rd, Meola G., Udd B., Ricker K. Report of the 84th ENMC workshop: PROMM (proximal myotonic myopathy) and other myotonic dystrophy-like syndromes: 2nd workshop. 13-15th October, 2000, Loosdrecht, The Netherlands. Neuromuscul. Disord., 12: 306-17, 2002.

Ono S., Kurisaki H., Inouye K., Mannen T. Raggedred fibers in myotonic dystrophy J. Neurol. Sci., 74: 247-55, 1986.

Ørngreen M.C., Olsen D.B., Vissing J. Aerobic Training in Patients with Myotonic Dystrophy Type 1. Ann. Neurol., 57: 754-757, 2005.

Pantic B., Trevisan E., Citta A., et al. Myotonic dystrophy protein kinase (DMPK) prevents ROSinduced cell death by assembling a hexokinase II-Src complex on the mitochondrial surface. Cell Death and Disease, 4: 1-11, 2013.

Peric S., Stojanovic V.R., Basta I., Peric M., Milicev M., Pavlovic S., Lavrnic D. Influence of multisystemic affection on health-related quality of life in patients with myotonic dystrophy type 1 . Clin. Neurol. Neurosurg., 115: 270-5, 2013. 
Reid S.F., Chalder T., Cleare A., Hotopf M., Wessely S. Chronic fatigue syndrome. BMJ Clin. Evid., 2008: 1101, 2008.

Reitan R.M. Validity of the Trail Making test as an indicator of organic brain damage. Percept. Mot Skills, 8: 271-276, 1958.

Sansone V.A., Panzeri M., Montanari M., Apolone G., Gandossini S., Rose M.R., Politano L., Solimene C., Siciliano G., Volpi L., Angelini C., Palmieri A., Toscano A., Musumeci O., Mongini T., Vercelli L., Massa R., Panico M.B., Grandi M., Meola G. Italian validation of INQoL, a quality of life questionnaire for adults with muscle diseases. Eur J Neurol., 17: 1178-87, 2010.

Sansone V.A., Ricci C., Montanari M., Apolone G., Rose M., Meola G., INQoL Group. Measuring quality of life impairment in skeletal muscle channelopathies. Eur. J. Neurol., 19: 1470-6, 2012.

Siciliano G., Manca M., Gennarelli M., Angelini C., Rocchi A., Iudice A., Miorin M., Mostacciuolo M. Epidemiology of myotonic dystrophy in Italy: re-apprisal after genetic diagnosis. Clin. Genet., 59: 344-9, 2001.

Søgaard K., Gandevia S.C., Todd G., Petersen N.T., Taylor J.L. The effect of sustained low-intensity contractions on supraspinal fatigue in human elbow flexor muscles. J. Physiol., 573: 511-523, 2006.

Spinnler H., Tognoni G. Standardizzazione e taratura italiana di test neuropsicologici. Ital. J. Neurol. Sci, 8: 1-120, 1987.

Taylor J.L., Gandevia S.C. A comparison of central aspects of fatigue in submaximal and maximal voluntary contractions. J. Appl. Physiol., 104 (2): 542-50, 1985.

Taylor J.L., Gandevia S.C. Transcranial magnetic stimulation and human muscle fatigue. Muscle Nerve., 24: 18-29, 2001.

Udd B. The myotonic dystrophies: molecular, clinical, and therapeutic challenges. Lancet Neurol., 11: 891-905, 2012.

Usuki F., Tagahashi N., Sasagawa N., Ishiura S. Differential signal pathways following oxidative stress in mutant Myotonin Protein Kinase cDNAtransfected C2C12 cell lines. Biochem Byophis Res Commun, 267: 739-43, 2000.

van Engelen B, OPTIMISTIC Consortium. Cognitive behaviour therapy plus aerobic exercise training to increase activity in patients with myotonic dystrophy type 1 (DM1) compared to usual care (OPTIMISTIC): study protocol for randomised controlled trial. Trials, 16: 224, 2015. 